\title{
Effects of Pulse Electroplating Parameters on Return Loss (S11) and Surface Roughness of Silver Coatings
}

\author{
Caner BAȘARAN ${ }^{\mathrm{a}, \mathrm{b}}$, İshak KARAKAYA ${ }^{\mathrm{a}}$ \\ aDepartment of Metallurgical and Materials Engineering, Middle East Technical University, \\ Ankara, Turkey \\ ${ }^{\mathrm{b}}$ REHIS, ASELSAN Inc., Ankara, Turkey
}

\begin{abstract}
1. Abstract
The highly conductive silver is widely used in the electronics industry, especially in microwave systems [1]. A silver-plated part will reduce loss at the highest microwave frequencies. In addition to this, it will carry extremely high current load. Surface roughness which distorts the propagation of electromagnetic waves is known to affect the uniformity of the current distribution of very good conductive metals such as silver [1]. The effects of duty cycle, frequency and average current density, which are pulsed silver-plating parameters, on the surface quality of coating have been investigated in this study. These effects have been examined by comparing the return loss (S11). S11 comparisons were made according to a reference gold plate. When S11 comparisons have been made, increasing return loss appears with increasing surface roughness. These effects have been characterized by SEM and XRD.
\end{abstract}

\section{Introduction}

It is known that although there were no patent for silver plating until 1840, silver plating has been applied since the $19^{\text {th }}$ century and mainly used to produce mirrors. Compared to other metals pure silver has the highest thermal and electrical conductivity. This property is also valid for the electroplated thin film forms. Therefore, many printed circuit systems include electroplated silver because of the highest thermal and electrical conductivity of silver among other metals [2]. Pulse wave rectifiers more expensive compared to DC rectifiers have been presented with the developments in electronics. Although, pulse techniques primarily were used in electronic industries, the advantages of this technique have been started to be observed in different industries. In addition to alloys, electrodeposition of single metals can also be performed by this technique. There are many application areas of pulse techniques like anodizing, amorphous alloys, semiconductor and composite plating [3,4,5,6]. DC plating is the mainly applied and known form of electrodeposition. This electrodeposition can be modified to pulse electrodeposition form by applying current interruption and reversal of current [7]. In contrast to pulse plating, when using direct current, there will be limited parameters. These parameters are current and potential. There are several other variables like duration, duty cycle and current of pulse in the case of pulse plating [8]. The porosity and rough deposits are the main undesirable forms of direct current 
deposition [9]. However, these limitations can be overcome by pulse application. In addition to these benefits, this technique enhances many properties which are not possible to gain by DC plating. The properties like lower porosity, ductility, hardness, finer grain size, electrical conductivity, plating thickness distribution, anti-tarnishing, coating life time improvement, immense saving in the consumption of raw material and enhancement in surface property may be achieved by pulse plating. In pulse electrodeposition, metal electroplating costs can be decreased by increasing current efficiency [10]. The pulse parameters are mainly consisted of three variables. The first one is peak current density, the second is current on time and the third one is current off time. The sum of on and off time related to one pulse cycle as shown in equation below. The coating with pulsating current seems more reasonable, considering the deposits in conventional coatings, yielding inhomogeneous thickness distributions, and the high costs to homogenize them [11]. The relationships for average current density, $i_{a}$, peak current density, $i_{p}$, and frequency are given below.

$$
\begin{aligned}
& \mathrm{i}_{a}=i_{p} * \text { duty cycle } \\
& \text { Frequency }=\frac{1}{\text { on time }+ \text { off time }} \\
& m=\frac{M * I * T}{F * N} \\
& m=\text { Theoretical yield (current efficiency) } \\
& M=\text { Molar mass (weight of displaced element in grams) } \\
& \text { I=Amperes } \\
& T=\text { Time in seconds } \\
& N=\text { Oxidation state (number of displaceable electrons per atom) } \\
& F=\text { Faraday's constant (96500 Coulombs) }
\end{aligned}
$$

In traditional DC and pulse electroplating average current density is similar. There are many alternatives of different pulse current densities and duty cycle range can be changed from 1 to $100 \%$. On and off times can also be varied from microseconds to milliseconds [12-13].

$$
\text { Duty cycle }=\frac{\text { on time }}{\text { on time }+ \text { off time }} * 100
$$

A typical pulse waveform shown in Figure 1 [15] where, on time is the cathodic time and average current density is the cathodic current density. One cycle is the sum of on time and off time. 


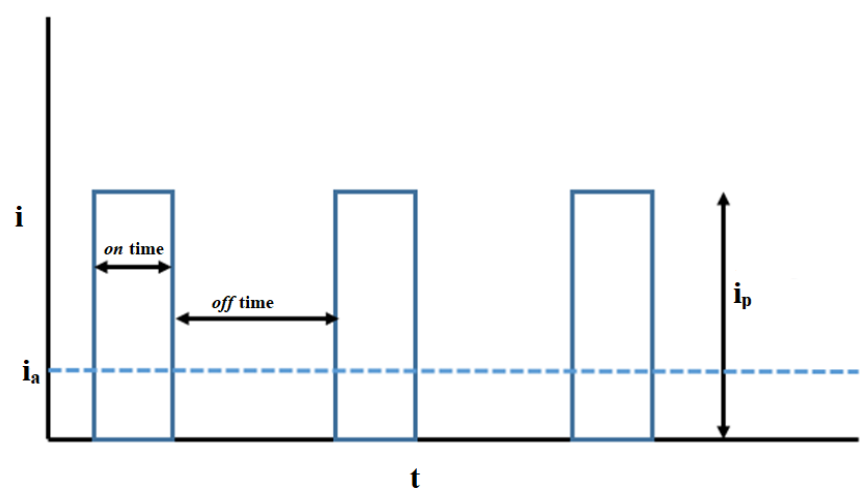

Figure 1: A typical pulse waveform

Coating quality depends on pulse parameters in pulse coating. Several morphologies of silver particles with several properties may be obtained by pulse current [14]. Therefore, these parameters should be optimized to make a better coating with a certain objective goal. The effects of pulse parameters were used to improve the return loss of the waveguides in this study [16]. Waveguides can be mentioned that having their high power carrying capacities, thermal stability, low loss and high quality factor so they are preferred in satellite communication, military applications and mobile communication systems. The performance of these waveguides can be compared with the S-parameter [16]. The input-output correlation among ports in an electrical framework decribes S-parameters. For example, when there are two ports ( Port 1 and Port 2), then S12 symbolizes transfering power from Port 2 to Port 1. So, S21 symbolizes the transfering power from Port 1 to Port 2 [15]. In fact S11 is the most commonly used parameter for wavequides. S11 exemplify the reflected amount of power from the wavequide, and therefore it can be accepted as the reflection coefficient or return loss. When $S 11=0 \mathrm{~dB}$, then all the power is reflected from the antenna and nothing is radiated. This can be considered as excellent conductivity only when the antenna is not considered as a whole [16]. For example, when S11=$5 \mathrm{~dB}$, this means that if $2 \mathrm{~dB}$ of power is given to the antenna, $-3 \mathrm{~dB}$ is the power that comes from reflecance [17]. The last amount of power is "absorbed" or given to the antenna. This accepted power is either radiated or absorbed as losses within the antenna. Antennas are usually designed to have low return loss. In this case, most of the power supplied to the antenna is emitted. Waveguides can also be considered similarly. Transmission and routing are important in these systems. The transmission must be at a minimum loss [17]. The scattering parameter was measured relative to a gold-plated sheet and the results were compared according to gold plate in this study. The relation between the scattering parameter and the coating quality will be examined in terms of surface roughness. The origin of all loss usually comes from the surface roughness, it has been observed that if roughness comes to the skin depth, attenuation of trasnsmission lines increase. 


\section{Experimental}

A typical cell used during silver electroplating can be seen elsewhere [18]. A solution containing only potassium and silver cyanide salts was used for this pulse plating. Brighteners were not added. Therefore, any organic addition was not made to attain white silver deposits. The presence of silver coating of antenna was sufficient since the coating was not applied for decorative purpose. Anti-tarnish coating for Silver Plating was applied to prevent atmospheric attack. Highpurity copper coupons were plated by changing the pulsed silver-plating parameters during experiments. The silver plated coupons were connected to the input of a waveguide which works at high-frequency so that the S11 could be measured. S11 were calculated according to the reference gold sheet. S11 measurements were done using a Vector Network Analyzer (VNA), which can plot S11 against frequency. The surfaces of all substrate coupons were prepared under identical conditions for comparison of surface roughness measurement after coating. The surface roughness was compared with scattering parameter measurements and the surface coatings were characterized by XRD and SEM in this research.

\section{Results and discussion}

Average current density is an important parameter of pulsed silver coating [19]. The average current density can be considered as the current density of the coating if it were made by direct current [19]. The average density is found by multiplying peak current density and the duty cycle. Therefore, the average current density will increase when the on time of the current increases at a constant peak current density. As illustrated in Figure 2, it is observed that when there is an increase in the average current density, frequency and duty cycle nucleation rate increases [20]. Furthermore, a fine-grained structure was achieved as the overpotential was increased due to higher average current density. In addition, increase in the number of nucleation sites, decreased the average grain size of coatings.
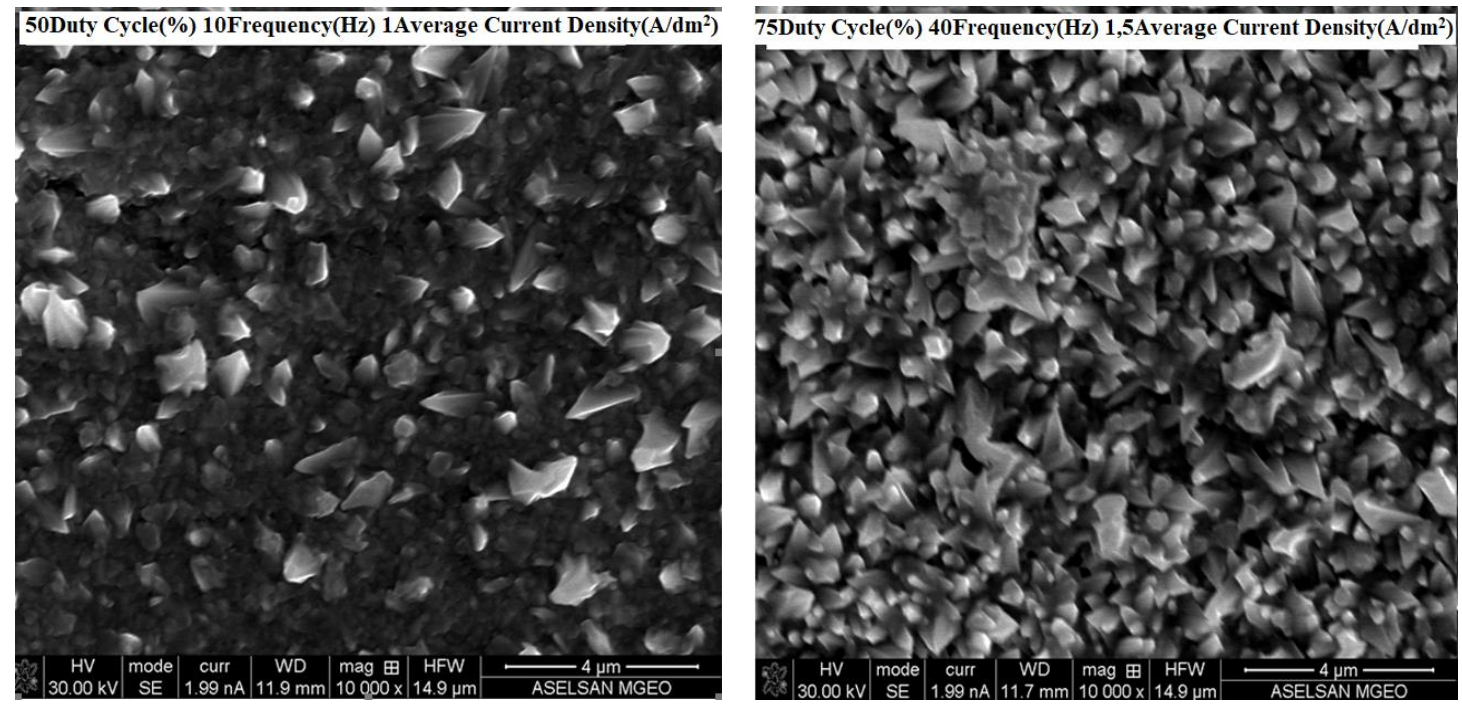

Figure 2: Grain structures of coatings at different pulse parameters 
The high average current density may provide a dendritic structure, but the frequency and duty cycle as well as the current density are important for controlling the dendritic growth [21]. On time and off time are effective about grain growth, because nucleation occurs during the on time and grain growth continues during the off time [21]. However, a raise in average current density at constant pulse charge and at constant off time leads to the reduction in grain size. For this reason, an inadequate on time hinders grain growth and enhances the nucleation rate. In order to understand the effect of frequency on grain structure, filling and unloading of the double layer should be considered [21]. There is not enough time for this double layer to fill or unload at high frequencies. When the frequency decreases, this double layer filling can be realized, and larger granular structures can be seen. Moreover, crystal structure is a native property of a material and cannot be altered. However, crystallite size and grain size are alterable, and depend on the production process [21]. All kinds of defects including grain boundaries restrict electron motion and negatively affect the conductivity. Usually, the electrical conductivity decreases with increasing porosity. Although pulse parameters may affect the conductivity by changing porosity and grain size, these effects may not be measured, or very sensitive devices are necessary to measure [21]. The surface roughness in the waveguide structures can be more effective in comparison to the transmission and spread of the material surface [16]. The grain size decrease when the frequency increases, as can be seen in Figure 2. Surface roughness value was $0.12 \mu \mathrm{m}$ at $\% 10$ duty cycle, $40 \mathrm{~Hz}$ frequency and $1.5 \mathrm{~A} / \mathrm{dm}^{2}$ average current density. It became $0.201 \mu \mathrm{m}$ at $\% 50$ duty cycle, $10 \mathrm{~Hz}$ frequency and $1 \mathrm{~A} / \mathrm{dm}^{2}$ average current density. Duty cycle reduces the surface roughness when frequency and average current density increase. Figure 3 shows the effect of three different pulse parameters on the reflection coefficient. It is observed that when grain structure of coating is homogenized, surface roughness decreases so return loss was decreased. It is also observed that the losses in the pulsed current are reduced compared to the direct current coating. As can be seen from Figure 3, when the surface roughness increased (no 2) the return loss was increased.

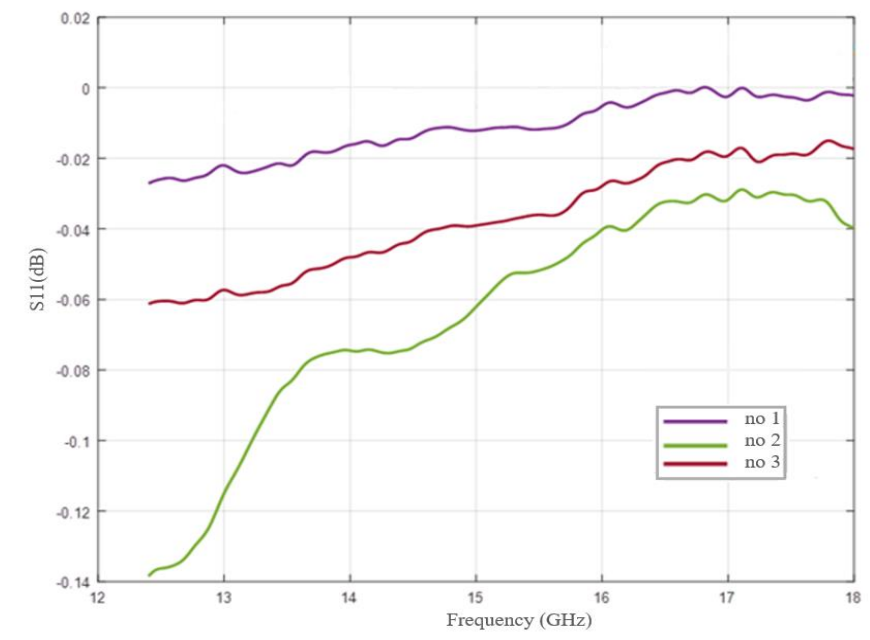

Figure 3: Return loss relative to the reference gold plate: $75 \%$ Duty Cycle, $40 \mathrm{~Hz}$ Frequency and $1.5 \mathrm{~A} / \mathrm{dm} 2$ Average Current Density (no 1); 100 \% Duty Cycle (no 2); 50 \% Duty Cycle, 10 Hz Frequency, and 1 A/dm2 Average Current Density (no 3) 


\section{Conclusions}

The effects of frequency, duty cycle, and average current density of pulse electroplating of silver on the roughness of the coating and return loss have been determined. It was observed that the surface roughness decreased when the duty cycle frequency and average current density was increased. In addition, the pulse parameters did not change the conductivity too much, but they affected the surface roughness and S11 scattering parameter. When surface roughness increases, return loss also increases. The results of the present work may be used to develop silver coating procedures to improve transmission quality of waveguides or reflector type antennas.

\section{References}

[1] Balanis, C. A. (1989). Advanced Engineering Electromagnetics. John Wiley \& Son

[2] Chandrasekar, M. S., \& Pushpavanam, M. (2008). Pulse and pulse reverse plating-Conceptual, advantages and applications. Electrochimica Acta, 53(8), 3313-3322. https://doi.org/10.1016/j.electacta.2007.11.054

[3] Osero, N. M. (1986). An overview of pulse plating. Plat. Surf. Finish., Vol. 73, pp. 20-22. Retrieved from http://www.dynatronix.com/wp-content/uploads/2014/02/Article-77.pdf

[4] Arslan, B., Demirci, G., Karakaya, İ. and Erdoğan, M. (2015). Formation of gold-plated electroformed copper structures. Advances in Materials and Processing Technologies, 1(3-4), pp. 384-393.

[5] Çetin, Y., Erdoğan, M., Demirci,. G., Karakaya,. İ. (2016). Nickel and Gold Coatings of Germanium and Kovar Substrates, ECS Transactions, $72 \quad$ (22) 55-64 http://dx.doi.org/10.1149/07222.0055ecst

[6] O. G. Göksu, M. Erdogan, and I. Karakaya, A Pulse Voltage Application in Electrochemical Reduction of Solid CaWO4 Powder, ECS Transactions, 72 (33) 31-39 (2016). http://dx.doi.org/10.1149/07233.0031ecst

[7] Seshadri, S. K. (1990). pulse plating review. Time, 25, 439-461.

[8] Devaraj, G., Guruviah, S., \& Seshadri, S. K. (1990). Pulse plating. Materials Chemistry and Physics, 25(5), 439-461. https://doi.org/https://doi.org/10.1016/0254-0584(90)90111-M

[9] Ashutosh Sharma, Siddhartha Das and Karabi Das (December 2nd 2015). Pulse Electroplating of Ultrafine Grained Tin Coating, Electroplating of Nanostructures, Mahmood Aliofkhazraei, IntechOpen, DOI: 10.5772/61255. Available from: https://www.intechopen.com/books/electroplating-of-nanostructures/pulse-electroplating-ofultrafine-grained-tin-coating 
[10] Randin, J.-P. (1988). Electrochemical assessment of the tarnish resistance of decorative gold alloys. Surface and Coatings Technology, 34(3), 253-275. https://doi.org/https://doi.org/10.1016/0257-8972(88)90117-X

[11] Randin, J.-P. (1988). Electrochemical assessment of the tarnish resistance of decorative gold alloys. Surface and Coatings Technology, 34(3), 253-275. https://doi.org/https://doi.org/10.1016/0257-8972(88)90117-X

[12] Kanani, N. (2004). Electroplating Basic principles: Processes and practice. Berlin: Elsevier Ltd.

[13] Chandrasekar, M. S., \& Pushpavanam, M. (2008). Pulse and pulse reverse platingConceptual, advantages and applications. Electrochimica Acta, 53(8), 3313-3322. https://doi.org/https://doi.org/10.1016/j.electacta.2007.11.054

[14] Durney, L.J.(1984). Electroplating Engineering Handbook. 4th ed. London: Chapman \& Hall.

[15] Ali SKIMA, M., KAROUI, M. S., Hamadi, G., \& Samet, M. (2006). S-PARAMETERS EXTRACTION OF A MULTI-PORT NETWORK USING PSPICE. https://doi.org/10.13140/2.1.1383.5520

[16] Pozer, D. (2005). Microwave Engineering Third Edition. John Wiley \& Son

[17] Hewlett-Packard Application Note 154. (1972). S Parameter Design. http://hparchive.com/appnotes

[18] F. Ulu, G. Demirci, M. Erdoğan, İ. Karakaya, The Role of Electrolyte Composition on Codeposited Ag and $\mathrm{Cu}$, ECS Transactions, 72 (21) 35-44 (2016). http://dx.doi.org/10.1149/07221.0035ecst.

[19] Arunachalam, R. M. (2009). Study of surface morphology in DC and pulse plating of silver alloy. 16(April), 128-132.

[20] Chandrasekar, M.S., Pushpavanam,M. (2008). Pulse and pulse reverse plating. Central Electrochemical Research Institute. 3313-3322

[21] Corresponding, C. S. (n.d.). Studies on the Effect of Base Metal Composition in Pulse Plating of Silver over Silver Alloy. 2(1), 14-18. 